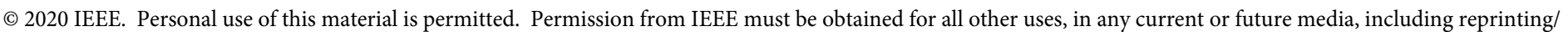

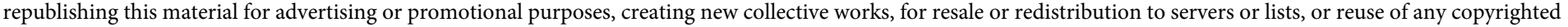
component of this work in other works.

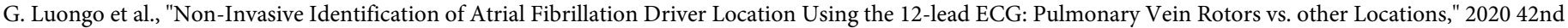
Conference of the IEEE Engineering in Medicine \& Biology Society (EMBC), Montreal, QC, Canada, 2020, pp. 410-413, doi: 10.1109/EMBC44109.2020.9176135.

\title{
Non-Invasive Identification of Atrial Fibrillation Driver Location Using the 12-lead ECG: Pulmonary Vein Rotors vs. other Locations
}

\author{
G. Luongo ${ }^{1}$, L. Azzolin ${ }^{1}$, M.W. Rivolta ${ }^{2}$, R. Sassi ${ }^{2}$, J.P. Martínez ${ }^{3}$, P. Laguna ${ }^{3}$, O. Dössel ${ }^{1}$, and A. Loewe ${ }^{1}$
}

\begin{abstract}
Atrial fibrillation (AF) is an irregular heart rhythm due to disorganized atrial electrical activity, often sustained by rotational drivers called rotors. In the present work, we sought to characterize and discriminate whether simulated single stable rotors are located in the pulmonary veins (PVs) or not, only by using non-invasive signals (i.e., the 12-lead ECG). Several features have been extracted from the signals, such as Hjort descriptors, recurrence quantification analysis (RQA), and principal component analysis. All the extracted features have shown significant discriminatory power, with particular emphasis to the RQA parameters. A decision tree classifier achieved $\mathbf{9 8 . 4 8 \%}$ accuracy, $\mathbf{8 3 . 3 3 \%}$ sensitivity, and $100 \%$ specificity on simulated data.
\end{abstract}

Clinical relevance - This study might guide ablation procedures, suggesting doctors to proceed directly in some patients with a pulmonary veins isolation, and avoiding the prior use of an invasive atrial mapping system.

\section{INTRODUCTION}

Atrial fibrillation (AF) is the most common sustained arrhythmia in clinical practice and a leading cause of hospitalization and death [1]. Rotors are localized functional reentrant circuits that sustain AF and exhibit curved wavefronts and wavetails that meet each other at a singularity point [2]. Ablation is one common therapy to terminate AF. Nevertheless, it is still uncertain which ablation approach is the most effective. Whether to ablate the "triggers" that initiate AF or the "substrate" that supports it. Narayan et al. showed how localization and ablation of rotors and focal sources is important to terminate AF [3], due to these mechanisms being drivers or organizing sources of fibrillation.

In clinical practice, pulmonary vein isolation (PVI) is one of the most common ablation methods that is applied to try to terminate AF. Since, triggers and sustaining mechanisms are often located on the pulmonary veins (PVs) [4]. In this work, we focused on identifying stable rotors that are located near to the PVs as opposed to other sites in the atria by using 12lead electrocardiogram (ECG) in a simulation study. The use of a non-invasive technique (i.e., 12-lead ECG) may directly suggest to the doctor if PVI alone will be successful or if additional procedures are likely required to terminate AF.

Research supported by the European Union's Horizon 2020 research and innovation programme under the Marie Skłodowska-Curie grant agreement No.766082 (MY-ATRIA).

${ }^{1}$ G. Luongo, L. Azzolin, O. Dössel, and A. Loewe are with the Institute of Biomedical Engineering (IBT), Karlsruhe Institute of Technology (KIT), 76131, Karlsruhe, Germany publications@ibt.kit.edu

${ }^{2}$ M.W. Rivolta, and R. Sassi are with the Dipartimento di Informatica, Università degli Studi di Milano, 20133, Milan, Italy

${ }^{3}$ J.P. Martínez, and P. Laguna are with the BSICoS group, Universidad de Zaragoza and with CIBER BBN, 50018, Zaragoza, Spain
In case of PVs rotor identification, the application of more complex and prior invasive mapping procedures could be avoided, proceeding directly with PVI.

\section{METHODS}

\section{A. Simulations}

The simulations were computed on a volumetric atrial geometry built from clinical data modelled with 11 million tetrahedral elements with fibre direction computed by a semiautomatic rule based algorithm [5]. Cellular atrial electrophysiology was represented by the Courtemanche-RamirezNattel model considering atrial fibrillation-induced remodelling in 9 regions with different conduction velocities [6] to take into account heterogeneity and anisotropy in the atria. The atrial geometry was considered with and without fibrotic tissue. Transmural fibrotic tissue was modelled as 2 circular patches with a radius of $14 \mathrm{~mm}$ in which $50 \%$ of the elements were not conductive and the other 50\% included ionic changes to represent the effect of cytokines (TGF- $\beta 1$ ) [7]. Single rotor (1R) episodes were induced using the phase singularity distribution method [8], which consists of placing phase singularities in the atria, estimating an activation time map by solving the Eikonal equation, and using this as initial state for a monodomain simulation [9]. The phase singularities were placed in 300 uniformly distributed points in the atria, and $3 \mathrm{~s}$ of activation were computed. Only the cases with $1 \mathrm{R}$ episodes that kept going for the whole simulation time were considered as stable rotor arrhythmias and considered for further analysis. This led to unbalanced data generation. From the transmembrane voltage (TMV) as the result of the monodomain simulation, the body surface potential map (BSPM) was calculated in 8 different torso models (19.898 triangles on average), generated from segmented MRI data of healthy male and female subjects (Fig. 1A.1-2, B.1-2), [10], [11]. The boundary element method was used to solve the forward problem of electrocardiography [12]. From the BSPM, the 12-lead ECG was extraced (Fig. 1A.3, B.3). Every 12-lead ECG signal has a length of $3 \mathrm{~s}$. The 12-lead ECG signals are formed only by f-waves without the QRS complex and T-wave, since the ventricles were not included in the simulations. The final dataset was composed of 440 simulated 12-lead ECGs (40, 112, and 288 ECGs with 1R located in the PVs, other left atrium areas, and right atrium areas, respectively).

\section{B. Feature Extraction}

100 features (Table I) were extracted from the 12-lead ECGs using biosignal processing methods: 
A.1

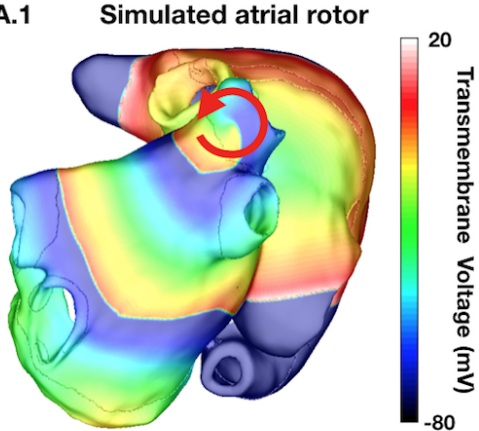

B.1 Simulated atrial rotor

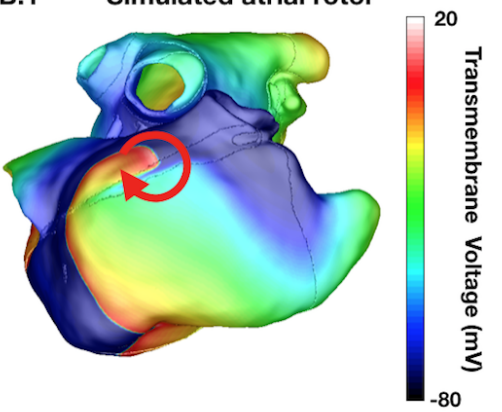

A.2

Body surface potential map

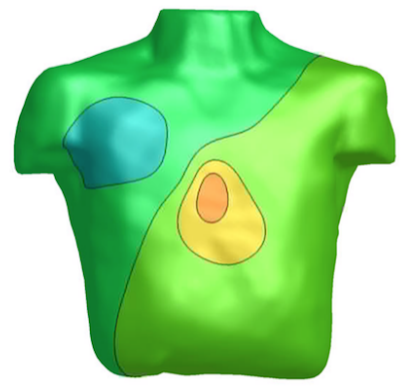

B.2

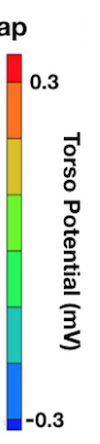

\section{ap}

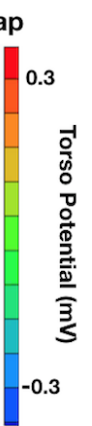

A.3 Electrocardiogram

(mV)
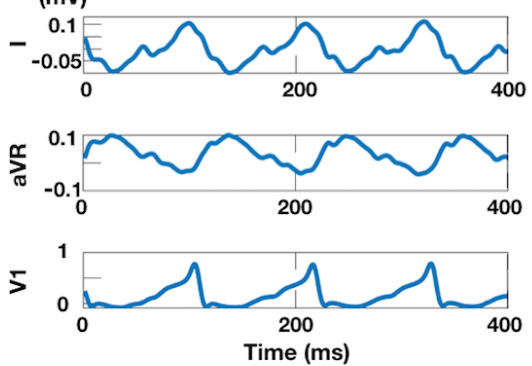

B.3

Electrocardiogram (mV)
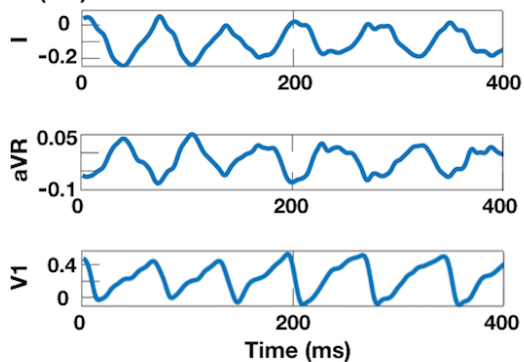

Fig. 1. A.1: Example of simulated atrial rotor located in the PVs. B.1: Example of simulated atrial rotor located outside the PVs (right atrium in this case). A.2-B.2: BSPMs on torso model generated from MRI. The torso potentials were obtained by solving the forward problem of electrocardiography from the simulated TMVs on the atria. A.3-B.3: Example of three of the 12 ECG leads extracted from the BSPMs.

TABLE I

FEATURE LIST

\begin{tabular}{|c|c|c|c|c|c|c|}
\hline 1. $\overline{\mathscr{H}_{0}}$ & 2. $\sigma_{\mathscr{H}_{0}}^{2}$ & 3. $\overline{\mathscr{H}_{1}}$ & 4. $\sigma_{\mathscr{H}_{1}}^{2}$ & 5. $\overline{\mathscr{H}_{2}}$ & 6. $\sigma_{\mathscr{H}_{2}}^{2}$ & 7. $\mathscr{D}_{V C G}$ \\
\hline 8. $\mathscr{E}_{V C G}$ & 9. $\mathscr{R}_{V C G}^{R}$ & 10. $\mathscr{L}_{V C G}$ & 11. $\mathscr{T}_{V C G}$ & 12. $\mathscr{E}_{V C G}$ & 13-14. $\mathscr{D}_{r l s R Q A_{d}}$ & 15-16. $\mathscr{E}_{r l s R Q A_{d}}$ \\
\hline 17-18. $\mathscr{R}_{r l s R Q A_{d}}^{R}$ & 19-20. $\mathscr{L}_{r l s R Q A_{d}}$ & 21-22. $\mathscr{T}_{r l s R Q A_{d}}$ & 23-24. $\mathscr{E}_{r l s R Q A_{d}}$ & 25-28. $\mathscr{R}_{s R Q A_{P C i}}^{R}$ & 29-32. $\mathscr{D}_{S R Q A_{P C i}}$ & 33-36. $\mathscr{L}_{S R Q A_{P C i}}$ \\
\hline 37-40. $\mathscr{T}_{s R Q A_{P C i}}$ & 41-44. $\mathscr{E}_{S R Q A_{P C i}}$ & 45-48. $\mathscr{E}_{S R Q A_{P C i}}$ & $49-60 . \overline{\lambda_{i}}$ & 61-72. $\sigma_{\lambda_{i}}$ & 73. $\bar{\lambda}_{P C}$ & 74. $\sigma_{\lambda_{P C}}$ \\
\hline 75-86. $\bar{R}_{i}$ & 87-98. $\sigma_{R_{i}}$ & 99. $\bar{R}_{P C}$ & 100. $\sigma_{R_{P C}}$ & & & \\
\hline
\end{tabular}

1) Hjort descriptors: The Hjort descriptors are closely related to the spectral moments. The first descriptor, $\mathscr{H}_{0}$, called activity, is defined by the total signal power. The second descriptor, $\mathscr{H}_{1}$, called mobility, reflects the dominant frequency of the signal under analysis. The third descriptor, $\mathscr{H}_{2}$, is used to define a measure related to half of the bandwidth of the signal and is termed complexity [16]. These descriptors were evaluated for each ECG lead. The mean values and the variances of these parameters over all 12 leads were calculated and used as features (feat. 1-6 in Table I).

2) Recurrence quantification analysis on vectocardiogram: The vectocardiogram (VCG) was calculated from the 12-lead ECG using the Dower's inverse transformation, and the 3-D VCG vector loops were used as state space plots for a further recurrence quantification analysis (RQA) [13]. RQA allowed to analyse the topological structure of multidimensional dynamical systems, giving access to a signal's intermittency, regularity, and predictability [14]. A detailed explanation of the RQA and the respective extractable parameters can be found in the work of Marwan et al., [14]. The extracted parameters were: determinism $\left(\mathscr{D}_{V C G}\right)$, entropy of the diagonal lines $\left(\mathscr{E}_{V C G} D L\right)$, recurrence rate $\left(\mathscr{R}_{V C G}^{R}\right)$, laminarity
$\left(\mathscr{L}_{V C G}\right)$, trapping time $\left(\mathscr{T}_{V C G}\right)$, and entropy of the vertical lines $(\mathscr{E} V L L$ ), (feat. 7-12 in Table I).

3) reduced lead spatial RQA: From the 12-lead ECGs, the first four principal components (PCs) were extracted (representing more than $99 \%$ of the total variability). The first three PCs, and the first four PCs, were used as dimensions (d) of a state space in which a reduced-lead spatial RQA $\left(r l s R Q A_{3}\right.$ and $\left.r l s R Q A_{4}\right)$ was applied, respectively [15]. The extracted parameters were: determinism $\left(\mathscr{D}_{\text {rls }_{S} Q A_{d}}\right)$, entropy of the diagonal lines $\left(\mathscr{E}_{r l s R Q A_{d}}\right)$, recurrence rate $\left(\mathscr{R}_{r l s R Q A_{d}}^{R}\right)$, laminarity $\left(\mathscr{L}_{r l s R Q A_{d}}\right)$, trapping time $\left(\mathscr{T}_{r l s R Q A_{d}}\right)$, and entropy of the vertical lines $\left(\mathscr{E} V L L R Q A_{d}\right)$, (feat. 13-24 in Table I).

4) standard RQA: A standard RQA (sRQA) was also applied on each of the first four PCs calculated from the 12-lead ECGs [15]. The extracted parameters were: determinism $\left(\mathscr{D}_{S R Q A_{P C i}}\right)$, entropy of the diagonal lines $\left(\mathscr{E}_{S R Q A_{P C i}}\right)$, recurrence rate $\left(\mathscr{R}_{S R Q A_{P C}}^{R}\right)$, laminarity $\left(\mathscr{L}_{S R Q A_{P C i}}\right)$, trapping time $\left(\mathscr{T}_{S R Q A_{P C}}\right)$, and entropy of the vertical lines $\left(\mathscr{E}_{S R Q A_{P C i}}\right)$, with $i$ being the number of PC, (feat. 25-48 in Table I).

5) Ratio PCA eigenvalues: The 12-lead ECGs were divided in 3 segments of the same length (i.e., $1 \mathrm{~s}$ considering the total length of each ECG of $3 \mathrm{~s}$ ). For each segment 
$j$, the eigenvalues $\left(\lambda_{i, j}\right)$ corresponding to the spatial PCA components over the 12 leads were extracted. From the $\lambda_{i, j}$, also the ratio was calculated:

$$
R_{i, j}=\frac{\lambda_{i, j}}{\sum_{k \neq i} \lambda_{k, j}}
$$

with $k$ being the number of PC.

The features extracted were: the mean $\lambda$ values and the respective standard deviations for each PC over all segments $\left(\bar{\lambda}_{i}\right.$ and $\left.\sigma_{\lambda_{i}}\right)$; from $\overline{\lambda_{i}}$, the mean over the 12 PCs and the respective standard deviation $\left(\bar{\lambda}_{P C}\right.$ and $\left.\sigma_{\lambda_{P C}}\right)$; the mean $\mathrm{R}$ values and the respective standard deviations for each PC over all segments $\left(\bar{R}_{i}\right.$ and $\left.\sigma_{R_{i}}\right)$; from $\bar{R}_{i}$, the mean over the $12 \mathrm{PCs}$ and the respective standard deviation $\left(\bar{R}_{P C}\right.$ and $\sigma_{R_{P C}}$ ), (feat. 49-100 in Table I). The idea behind the $\mathrm{R}_{i, j}$ parameter and the extracted features was to increase the differences between the eigenvalues to achieve a better discrimination due to the variability shown by the PCs over time and between them.

\section{Feature Selection}

Features were selected with a greedy forward selection technique to implement a feature set. This algorithm started with an empty feature set and added a new feature to it at each iteration based on the increase of the feature set accuracy with a decision tree classifier. The algorithm stopped when performance based on the validation set could not be further increased. In order to handle possible correlations among features, the candidate feature to be added to the set was only added if the correlation coefficient with any of the already included features was $<0.6$. The correlation threshold was optimized looking for the best compromise between redundant information and physiological explanation.

\section{Classification}

In this preliminary study, a decision tree classifier was implemented for binary classification (1R located in PVs vs. noPVs) due to its simplicity. All extracted features were singularly evaluated with a simple decision tree classifier and a leave-one-out cross-validation procedure. Subsequently, a multi-feature classification was performed with the feature set selected in section II-C. The dataset was randomly divided in training set, validation set, and test set with a ratio of $70 \%, 15 \%$, and $15 \%$, respectively. Training set was used for the tuning of classifier parameters, while validation set was used for the greedy feature selection optimization. The classes have been balanced appropriately weighting them in the dataset. Sensitivity and specificity were calculated considering the class PVs rotors as positive and the class noPVs rotors as negative.

\section{E. Statistical Analysis}

The evaluation of goodness of each single feature in characterizing the two classes was done with the Wilcoxon rank-sum test. p-values $<0.01$ were considered statistically significant.
TABLE II

5 SINGLE FEATURES WITH THE HIGHEST ACCURACY FOR PVS VS. NOPVS CLASSIFICATION

\begin{tabular}{lccc}
\hline Feature & Accuracy $(\%)$ & Sensitivity $(\%)$ & Specificity (\%) \\
\hline $\mathscr{L}_{V C G}$ & 95.23 & 80.00 & 96.75 \\
$\mathscr{D}_{r l s R Q A_{3}}$ & 94.55 & 80.00 & 96.00 \\
$\bar{R}_{P C}$ & 93.86 & 75.00 & 95.75 \\
$\mathscr{E}_{\text {rls }}$ & 93.41 & 75.00 & 95.25 \\
$\mathscr{E}_{V C L}$ & 93.18 & 75.00 & 95.00 \\
\hline
\end{tabular}
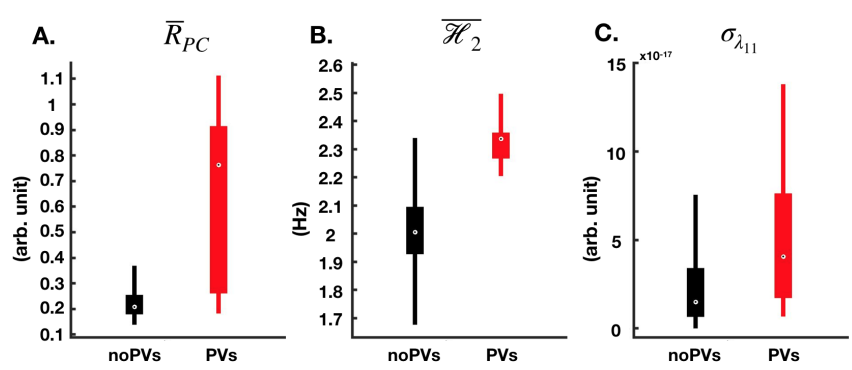

Fig. 2. Boxplots for PVs vs. noPVs rotor location classification: A. $\bar{R}_{P C}$ feature, B. $\overline{\mathscr{H}}_{2}$ feature, C. $\sigma_{\lambda_{11}}$ feature. All features are statistically different between the two classes with $\mathrm{p}<0.01$.

\section{RESULTS}

\section{A. Characterization of PVs Rotors}

The 5 features with the highest accuracy for individually discriminating these phenomena are shown in Table II. All these features showed significantly higher values for $1 \mathrm{R}$ located in the PVs with $\mathrm{p}<0.01$.

\section{B. PVs Rotors Classification}

The feature set comprised 3 features: $\bar{R}_{P C}, \overline{\mathscr{H}}_{2}$, and $\sigma_{\lambda_{11}}$. As shown in Fig. 2, these 3 features were significantly higher for $1 \mathrm{R}$ located in the PVs. The classifier reached a test accuracy of $98.48 \%$, with sensitivity of $83.33 \%$, specificity of $100 \%$, and negative predicted value of $98.36 \%$.

\section{DISCUSSION}

The simulations implemented in this work provide ideal and controlled scenarios where the ground truth for AF perpetuation sustained by $1 \mathrm{R}$ is known in all the cases. This allows the analysis of each simulation without the influence of secondary, or unknown, mechanisms, e.g. other simultaneous rotors.

\section{A. Characterization of PVs Located Rotors}

There are compelling results for RQA approaches regarding characterization and discrimination of many arrhythmias [13], [15]. 4 of the 5 single highest ranked features in this work were obtained with RQA methods (Table II). We observed that the irregular activity produced by $1 \mathrm{R}$ located in the PVs is delimited in a small portion of tissue, due to the presence of many anatomical obstacles (i.e., the PVs). In fact, in our simulated cases, PVs prevent the rotor from meandering in the remaining areas of the LA. Therefore, the signal can propagate as a single wave front in the 
remaining atrial areas. This explains why, the determinism and laminarity of the ECG is significantly higher for PVs $1 \mathrm{R}$ cases. On the contrary, if the rotor is positioned in other atrial areas, it has less anatomical constraints and is free to meander throughout the tissue. This yields more irregular ECGs, therefore less deterministic and laminar. Entropy of the diagonal/vertical lines reflects the complexity of the phenomena regarding the determinism/laminarity. Hence, the same considerations made for determinism and laminarity are valid for diagonal and vertical entropy as well.

\section{B. PVs vs. noPVs 1R Classification}

The high performance achieved for PVs vs. noPVs 1R classification indicates the potential of using the features extracted in this work to identify the location of $1 \mathrm{R}$ (PVs vs. noPVs) using only the non-invasive 12-lead ECG signals.

The ECG produced by the PVs simulations is more regular than the one in the noPVs simulations, and a large amount of information can be contained in the PC1 in case of regular signals. As result of it, the $\mathrm{R}$ parameters calculated from the PC1 of PVs cases was considerably higher than the ones in noPVs cases. On the contrary, in the following PCs, the R parameters have a lower difference between the two classes, even though they are still higher in the PVs cases. This strong contribution from PC1 influences the $\bar{R}_{P C}$ feature, which becomes significantly higher in the PVs class than in the noPVs class (Fig. 2A.).

Complexity parameters have lower values if the signals under analysis are as sinusoidal as possible. In case of PVs simulations, the PCs are more regular and stable than in noPVs simulations (as confirmed by the RQA parameters). However, in the PVs case, the signals are less sinusoidal than those composing the signal in the noPVs case (Fig. 1A.3B.3). For this reason, $\overline{\mathscr{H}}_{2}$ is significantly higher for the PVs class than for the noPVs class (Fig. 2B.).

In case of regular signals, most of the variability is contained in the first PCs. On the contrary, the last PCs no longer contain much information, becoming irregular signals. Therefore, $\sigma_{\lambda}$ over each of these last PCs (i.e., $\sigma_{\lambda_{11}}$ ) for the PVs cases is significantly higher than in the noPVs cases (In Fig. 2C.).

The specificity of $100 \%$ indicates that the classifier was able to identify all the noPVs $1 \mathrm{R}$ in this work. Therefore, the classifier correctly predicted all cases where pure PV cryo-ablation would not be sufficient. Since, the $1 \mathrm{R}$ is not located on the PVs, and so the PVI will likely not stop the arrhythmia. For these cases, radio frequency ablation catheters and a prior mapping system are needed, to proceed with a more complex ablation procedure. Consequently, doctors could plan the procedure and equipment accordingly to the prediction made by the implemented classifier.

This work could be extended with a prior characterization of different AF driver mechanisms and AF complexity analysis. Several and more robust classification algorithms can be tested.

\section{CONCLUSIONS}

The parameters extracted from the RQA (sRQA, rlsRQA and RQA on the VCG) have shown to be the most performing in individually characterizing and discriminating whether $1 \mathrm{R}$ is located in the PVs or not, reaching 95\% accuracy even with a single feature classification.

A decision tree classifier was implemented with a feature set of 3 features yielding an accuracy of $98.48 \%$, specificity of $100 \%$, and a sensitivity of $83.33 \%$. This classifier could guide the doctors before the ablation procedures, suggesting if PVI could potentially terminate the ongoing arrhythmia or not, without the need of a prior invasive mapping procedure. Due to the computational framework of this study, further investigations on clinical data, exactly labelled by inspecting the local activation maps, are necessary to effectively assess the proposed approach.

\section{REFERENCES}

[1] H. Calkins et al., "2012 HRS/EHRA/ECAS expert consensus statement on catheter and surgical ablation of atrial fibrillation: recommendations for patient selection, procedural techniques, patient management and follow-up, definitions, endpoints, and research trial design," Heart Rhythm, vol. 9, no. 4, pp. 632-696, 2012.

[2] SV. Pandit and J. Jalife, "Rotors and the dynamics of cardiac fibrillation," Circ. Res., vol. 112, pp. 849-862, 2013.

[3] S.M. Narayan et al., "Ablation of rotor and focal sources reduces late recurrence of atrial fibrillation compared to trigger ablation alone," J Am Coll Cardiol., vol. 63, no. 17, pp. 1761-1768, 2014.

[4] M. Haissaguerre et al., "Spontaneous initiation of atrial fibrillation by ectopic beats originating in the pulmonary veins," N Engl J Med, vol. 339, pp. 659-666, 1998.

[5] A. Wachter et al., "Mesh structure-independent modeling of patientspecific atrial fiber orientation," CDBME, vol. 1, pp. 409-412, 2015.

[6] A. Loewe, "Chronic AF induced remodeling," in Modeling Human Atrial Patho-Electrophysiology From Ion Channels to ECG : Substrates, Pharmacology, Vulnerability, and P-Waves, Karlsruhe, Germany: KIT Scientific Publishing, 2016, ch. 5.2, pp. 113-119.

[7] C. H. Roney et al., "Modelling methodology of atrial fibrosis affects rotor dynamics and electrograms," Europace, vol. 18, pp. iv146-iv155, 2016.

[8] V. Jacquement., "An eikonal approach for the initiation of reentrant cardiac propagation in reaction-diffusion models," IEEE Trans. Biomed. Eng., vol. 57, pp. 2090-2098, 2010.

[9] E. J. Vigmond et al., "Computational tools for modeling electrical activity in cardiac tissue," J Electrocardiol, vol. 36, pp. 69-, 2003.

[10] M. W. Krueger et al., "Personalization of atrial anatomy and electrophysiology as a basis for clinical modeling of radio-frequency ablation of atrial fibrillation," IEEE Trans. Med. Imaging, vol. 32, no. 1, pp. 73-84, Jan. 2013.

[11] R.L. Lux et al., "Body Surface Potential Mapping Techniques," in Comprehensive Electrocardiology, London, UK: Springer, 2010, pp. 1361-1374.

[12] M. Stenroos, "The transfer matrix for epicardial potential in a piecewise homogeneous thorax model: The boundary element formulation," Phys. Med. Biol., vol. 54, no. 18, pp. 5443-55, 2009.

[13] H. Yang, "Mulstiscale recurrence quantification analysis of spatial cardiac vectorcardiogram signals," IEEE Trans. Biomed. Eng., vol. 58, no. 2, pp. 339-347, 2011.

[14] N. Marwan et al., "Recurrence plots for the analysis of complex systems," Physics Reports, vol. 438, no. 5-6, pp. 237-329, 2007.

[15] G. Luongo et al., "Non-Invasive Characterization of Atrial Flutter Mechanisms Using Recurrence Quantification Analysis on the ECG: a Computational Study," IEEE Trans. Biomed. Eng., accepted.

[16] L. Sörnmo and P. Laguna, "EEG Signal Processing," in Bioelectrical signal processing in cardiac and neurological applications, Burlington: Academic Press, 2005, ch. 3, section 3.3, pp. 100-102. 